Letter to the Editor

\title{
Outpatient Management of Patients in Singapore's National Centre for Infectious Diseases Special Precautions Area Clinic in the COVID-19 Pandemic
}

\section{Dear Editor,}

COVID-19 first entered public awareness on 29 Dec 2019, when the first four cases were reported in Wuhan, China. ${ }^{1}$ It has since become a global pandemic. The first case of COVID-19 in Singapore was confirmed on 23 Jan $20 .^{2}$ Applying lessons learned from the 2003 Severe Acute Respiratory Syndrome (SARS) experience, there has been a coordinated response across various ministries to i) identify suspected cases and put them under quarantine, ii) screen and diagnose those with COVID-19 early and keep them isolated until they are deemed to be no longer infective, and iii) implement national policies to prevent the import of new cases as well as local transmission to halt the spread of the Severe Acute Respiratory Syndrome Coronavirus 2 (SAR-CoV-2). ${ }^{3,4}$ These measures helped to limit community transmission of the virus but failed to completely contain it. While both the SARS-CoV-1 (responsible for SARS) and -2 viruses are members of the Coronaviridae family, a key difference is that SARS-CoV-2 exhibits infectivity early prior to symptoms developing and seems to have greater transmissibility. ${ }^{5,6}$

\section{The Special Precautions Area (SPA) Clinic}

The Special Precautions Area (SPA) is an area within the Infectious Diseases Outpatient Clinic where suspected cases of COVID-19 are seen. It is separate from the regular clinic area to prevent intermingling of potentially infective patients with the general patient population. It has a comprehensive suite of on-site

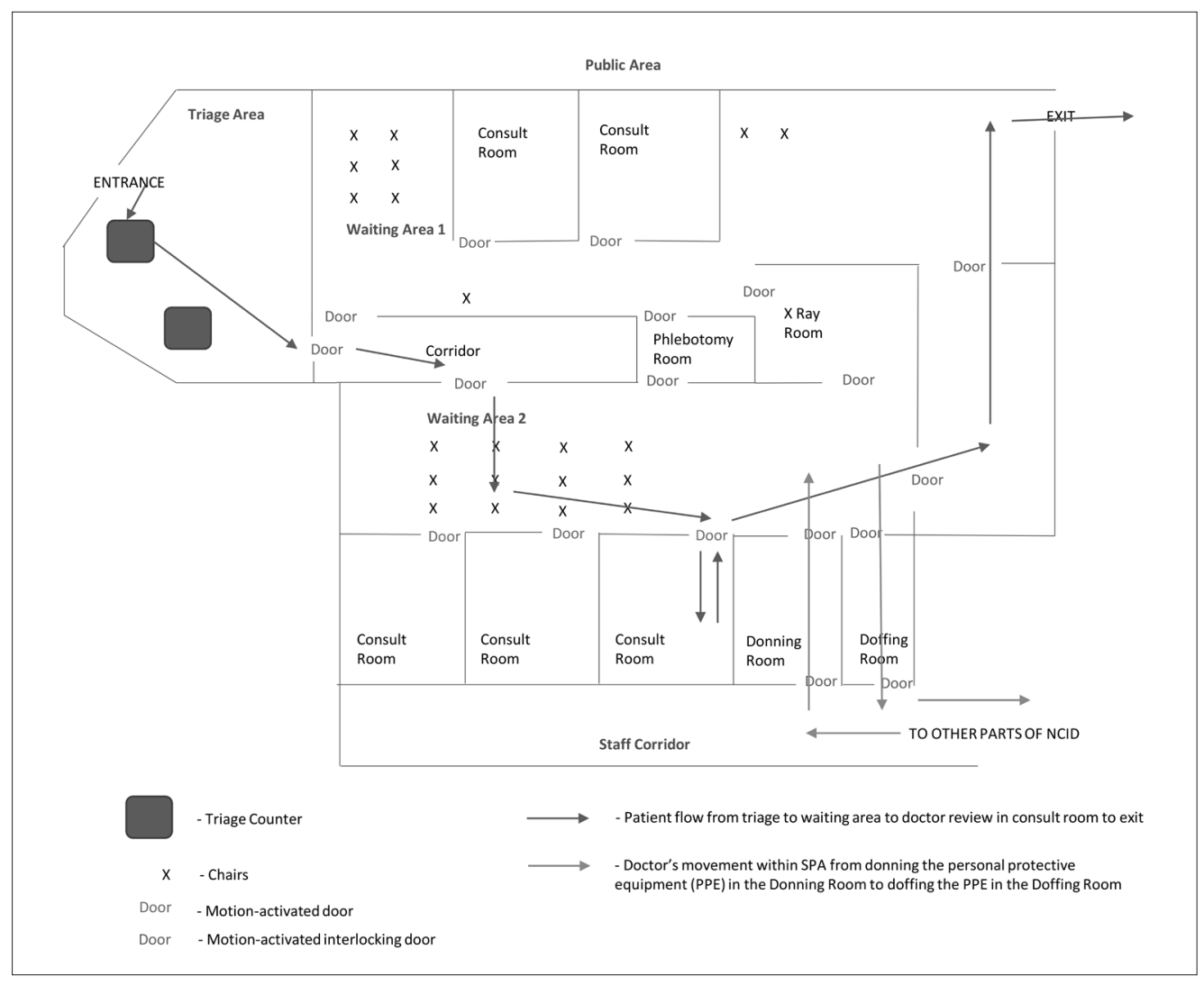


capabilities including staff to collect swab specimens, phlebotomy, and X-ray services. Infection control measures are implemented such as the requirement for all healthcare workers to don full personal protective equipment (PPE) before entering the area, negative pressure rooms for donning and doffing, and clear workflows to allow all staff to work safely. This was designed to prevent any potential transmission of COVID-19 to hospital staff working there and other patients who visit the clinic. ${ }^{7}$

In the initial period of the outbreak when the caseload was low, all patients who fulfilled the suspect criteria for COVID-19 infection were admitted into the National Centre for Infectious Diseases (NCID). Patients had to have 2 respiratory samples, collected 24 hours apart, negative for SARS-CoV-2 to be discharged. Patients who required a follow-up review (such as those who were still symptomatic, who were still early in their illness, or those with blood tests or chest radiograph abnormalities) would be seen in the SPA clinic.

As the caseload increased, to better rationalise inpatient resources, the NCID Screening Centre (SC) introduced a swab-and-discharge protocol for low-risk suspect patients (those who were clinically well and without evidence of pneumonia). ${ }^{8}$ Large numbers of suspect patients seen at SC daily were swabbed and discharged with a follow-up appointment at the SPA clinic for review of symptoms and consideration of repeat swab or further evaluation if required.

Confirmed COVID-19 patients, who were discharged after 2 respiratory samples 24 hours apart tested negative for SARS-CoV-2, were also given follow up appointments in the SPA clinic for review.

The SPA clinic saw a total of 1562 cases between 28 Jan 2020 and 23 Mar 2020, averaging about 40 cases per day. With the number of COVID-19 cases in Singapore crossing the 1000 mark, the workload in SPA is expected to increase further. Clear workflows are therefore important to maintain efficiency and safety in running the clinic. ${ }^{9}$

\section{The Development of the Defaulters Protocol}

One such workflow is the management of defaulters patients who do not turn up for their appointments. In the context of TB, patients who are lost to follow up (LTFU) are a unique clinical and epidemiological problem and is associated with poorer consequences and can also lead to outbreaks. ${ }^{10,11}$ While patients who are LTFU has not been well-studied in the context of novel virus outbreaks such as that of COVID-19, there are similar concerns that potentially infectious individuals can lead to outbreaks in the community if they are not diagnosed early.

These concerns have to be balanced against overloading the healthcare systems, as some do not show up because their symptoms have resolved while others may still exhibit mild symptoms and feel that they do not require follow-up. Given the large number of patients screened for COVID-19 in Singapore, there was a need to manage scarce outpatient slots for those who need them most. The average defaulter rates in the SPA clinic can go up to as high as $67 \%$. To this end, a telemedicine protocol (Figure 1) was implemented to manage these patients. The protocol was designed with three guiding principles in mind: (1) Accurate risk stratification, (2) Proper siting of care, (3) Establishment of a safety net to ensure that all patients who require an evaluation are seen.

\section{Accurate Risk Stratification}

The most important principle in the management of defaulters is to assess the likelihood of COVID-19 infection, as well as the risk of onward transmission. Confirmed cases are deemed to pose the highest risk since transmission dynamics are uncertain and following up with these patients is critical to better understand the risk of onward transmission. For those who are not confirmed cases, high-risk features include a patient who (1) had been discharged from the wards, (2) had travelled to areas of heightened vigilance as defined by Ministry of Health, Singapore, (3) had contact with a confirmed COVID-19 case, (4) had contact with a local cluster of COVID-19 cases. $^{12}$

\section{Proper Siting of Care}

After risk assessment, proper siting of care is possible (Figure 2). Patients who are high risk and whose symptoms are not improving will be prioritised for rescheduling of their clinic appointments (GX and G2). Those who are low-risk whose symptoms are not improving are also required to return (G3), but should they prefer not to, they will be directed to Public Health Preparedness Clinics (PHPCs) for review to focus hospital resources on the higher-risk patients. ${ }^{13}$

Patients who are uncontactable despite the best efforts by the Medical Officer (MO) or the Patient Service Associate (PSA) pose a significant challenge. Nevertheless, risk stratification will allow us to confidently manage these patients. Should a patient be deemed to be high risk but are uncontactable, further escalation up the chain of command will be necessary (G4). This has however not occurred thus far in the SPA clinic. Those who are low risk will be discharged (G5). 


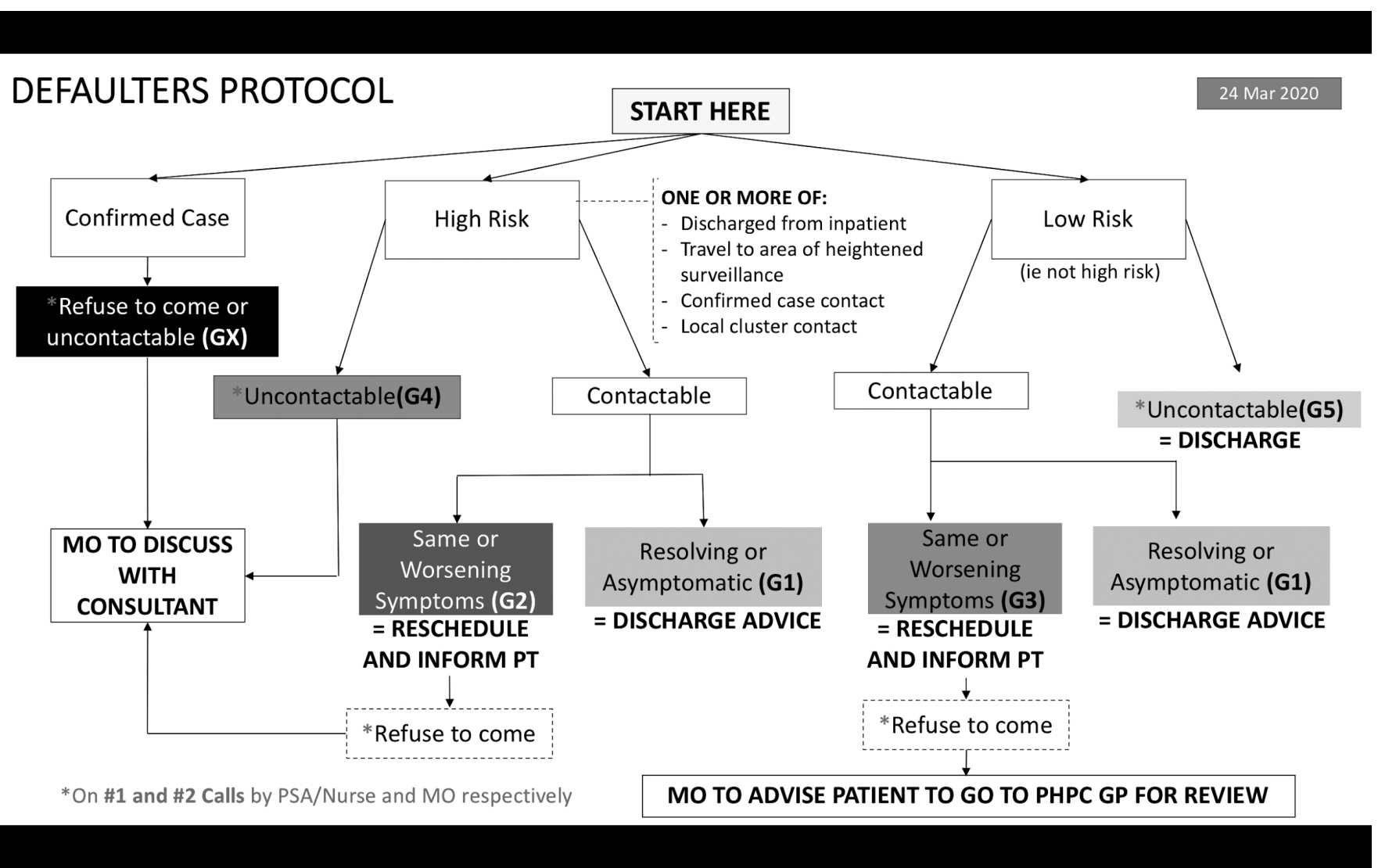

\section{Establishing Safety Nets}

Despite the above, there may be people who slip through the cracks. Recent research has shown that infectious COVID-19 patients may be asymptomatic or may have mild symptoms. ${ }^{14}$ Additionally, testing negative for COVID-19 may lull patients into a sense of security; they may still get the disease should they be exposed subsequently. As such, clear discharge advice was reiterated to patients who had resolving symptoms (G1) to return to the Screening Centre or PHPCs should they develop new symptoms. They are also encouraged to practice social distancing and good personal hygiene. With PHPCs located across the country, they serve as another important safety net to ensure that those with changes in their clinical condition can get quick access to care.

\section{Conclusion}

The SPA Clinic serves an important role in screening and evaluating large numbers of suspects in an outpatient setting, and allows us to save inpatient resources for those who need it most. In our bid to contain COVID-19 it is crucial to ensure that patients do not get lost to follow up. The principles used in the design of the telemedicine protocol could be helpful in situations where healthcare resources are taxed and the rationing of resources for severe cases is required.

\section{REFERENCES}

1. Zhu N, Zhang D, Wang W, et al. A novel coronavirus from patients with pneumonia in China, 2019. N Engl J Med 2020;382:727-733.

2. Abdullah X, Salamat H. Singapore confirms first case of Wuhan virus [Internet]. Channel News Asia. 2020 [cited 2020Apr2]. Available from: https://www.channelnewsasia.com/news/singapore/wuhan-viruspneumonia-singapore-confirms-first-case- 12312860 .

3. Tan CC. SARS in Singapore - Key lessons from an epidemic. Ann Acad Med Singap 2006;35:345-349.

4. Wong JEL, Leo YS, Tan CC. COVID-19 in Singapore-Current Experience Critical Global Issues That Require Attention and Action. JAMA 2020;323:1243-1244.

5. Li C, Ji F, Wang L, Wang L, Hao J, Dai M, et al. Asymptomatic and human-to-human transmission of SARS-CoV-2 in a 2-family cluster, Xuzhou, China. Emerg Infect Dis 2020; 26:1626-1628.

6. Wilder-Smith A, Chiew CJ, Lee VJ. Can we contain the COVID-19 outbreak with the same measures as for SARS? Lancet Infect Dis 2020;20:e102-e107.

7. Low JGH, Wilder-Smith A. Infectious respiratory illnesses and their impact on healthcare workers: a review. Ann Acad Med Singap 2005;34:105-10. 
8. Tan GSE, Ang H, Manauis CM, Chua JM, Gao CQ, Ng FKK, et al. Reducing hospital admissions for COVID-19 at a dedicated screening centre in Singapore. Clin Microbiol Infect 2020;26:1278-1279.

9. Yong C. 2020. Coronavirus cases in S'pore hit 1,000 with 74 new patients; 10 cases from old folks' home, including a 102-year-old. The Straits Times, [online] Available at: <https://www.straitstimes.com/ singapore/74-new-coronavirus-cases-in-spore-bringing-total-to-100054-new-cases-are-local-with-10>. Accessed on 2 April 2020.

10. Millet ERC, Noel D, Mangtani P, Abubakar I, Kruijshaar ME Factors associated with being lost to follow-up before completing tuberculosis treatment: Analysis of surveillance data. Epidemiol Infect 2013;141:1223-123.

11. Mitruka K, Blake H, Ricks P, Miramontes R, Bamrah S, Chee C, et al. A tuberculosis outbreak fueled by cross-border travel and illicit substances: Nevada and Arizona. Public Health Rep 2014;129:78-85.

12. MOH Singapore. 2020. Available from: https://www.moh.gov. $\mathrm{sg} / \mathrm{docs} /$ ibrariesprovider4/default-document-library/listof-countries areas-in-suspect-case-definition-(19mar20) d3ac51c163f54833a33f4810bcf0adeb.pdf.
13. MOH National Schemes [Internet]. AIC PCP. [cited 2020May28]. Available from: https://www.primarycarepages.sg/practice-management/ moh-national-schemes/public-health-preparedness-clinic-(phpc).

14. Gandhi M, Yokoe DS, Havlir DV. Asymptomatic Transmission, the Achilles' Heel of Current Strategies to Control Covid-19. N Engl J Med 2020;382:2158-2160.

Wilnard YT Tan, ${ }^{1}{ }_{M B B S}$, Gabrielle CXH Ng, ${ }^{1}{ }_{M B B S}$, Yun Yuan $\underline{\text { Mah, }},{ }^{1}$ MBBSS, Michelle CY Law, ${ }_{B S C}^{1}{ }_{\text {(Hons), }}$ MBBS, Cheng Seong Wong, ${ }^{2} M B B S, M R C P(U K)$

${ }^{1}$ Department of Medicine, Tan Tock Seng Hospital, Singapore

${ }^{2}$ National Centre for Infectious Diseases, Singapore

Address for Correspondence: Tan Yeong Tze Wilnard, Department of Medicine, Tan Tock Seng Hospital. 11 Jalan Tan Tock Seng, Singapore 308433 Email: wilnardtyt@gmail.com 